\title{
Evidence of Credit Risk Contagion Between Corporate and Financial Sectors ${ }^{*}$
}

\author{
Gabriel Gaiduchevici \\ Bucharest University of Economic Studies, Bucharest, Romania
}

\begin{abstract}
We propose a copula-GARCH framework to analyze the credit risk dependence structure between the European corporate and financial sectors. Empirical study is conducted on two representative iTraxx indices. In order to better capture the dynamic behavior of dependence we allow copula parameters to be time-varying by specifying them as a function of past values and standardized score of the copula log-likelihood. The results obtained indicate a time-varying dependence structure with statistically significant tail dependence coefficients supporting the existence of credit risk contagion effects. Goodness-of-fit tests are used to indicate which model is closest to the true conditional copula. Our findings provide further evidence supporting the interconnectivity of credit risk between corporates and financial institutions operating on the European market.
\end{abstract}

Keywords: credit risk, copula, contagion effects, goodness-of-fit tests, systemic risk, time-varying dependence

\section{Introduction}

We propose a framework for credit risk contagion analysis based on a time varying copula-GARCH type of model that allows for separate specification of the dependence structure from the marginal distributions. The empirical part analyses the credit risk dependence pattern between European corporate and financial sectors, as reflected by the iTraxx Senior Financial and Crossover CDS indices. These indices are highly representative aggregated indicators of credit risk in their respective sectors. In addition to flexibility, this set-up facilitates the credit risk analysis at sector level as well as the description of the dependence structure that leverages total risk.

The literature on copula modeling is growing by the day. For a thorough introduction to copulas we refer to Joe (1997) and Nelsen (2006), the two key text books on dependence modeling from a statistical perspective. McNeil, Frey, and Embrechts (2005) provided a sound implementation of copula models in the context of quantitative risk management while Embrechts, McNeil, and Straumann (2002) introduced the static representation of dependence via copulas. Patton (2006) laid down the foundation for multivariate financial time series applications of copulas and complements his research with a comprehensive empirical study in Patton (2012). Choroś, Ibragimov, and Permiakova (2010) presented an overview of copula estimation methods for time series data. Patton (2009) gave an overall survey of copula applications to time series. Genest,

\footnotetext{
* This work was co-financed from the European Social Fund through Sectoral Operational Programme Human Resources Development 2007-2013; project number POSDRU/107/1.5/S/77213, Ph.D. for a career in interdisciplinary economic research at the European standards. The scientific support and access to databases provided by Ladislaus von Bortkiewicz Chair of Statistics, C.A.S.E.-Center for Applied Statistics and Economics, Humboldt-Universitätzu Berlin is gratefully acknowledged. Gabriel Gaiduchevici, Ph.D. candidate, Money and Banking Department, Bucharest University of Economic Studies. Correspondence concerning this article should be addressed to Gabriel Gaiduchevici, 5-7 Moxa Street, district 1, 010961 Bucharest, Romania. E-mail: gaiduchevici@gmail.com.
} 
Rémillard, and Beaudoin (2009) and Rémillard (2010) are two key references for copula Goodness-of-Fit tests.

Sklar's theorem provides the formal approach to separate a joint distribution into independent margins and a copula. For every $p$-dimensional distribution $F$ with corresponding margins $F_{i}$, there exists a copula $C$ such that:

$$
F\left(y_{1}, \ldots, y_{p}\right)=C\left(F_{1}\left(y_{1}\right), \ldots, F_{p}\left(y_{p}\right)\right)
$$

If $F_{i}$ is continuous then the probability integral transformation $U_{i}=F_{i}\left(Y_{i}\right)$ is unique and Unif $(0,1)$ distributed regardless of the original distribution of $F_{i}$. Conversely:

$$
C\left(u_{1}, \ldots, u_{p}\right)=F\left(F_{1}^{-1}\left(u_{1}\right), \ldots, F_{p}^{-1}\left(u_{p}\right)\right)
$$

where $u_{i}=F_{i}\left(y_{i}\right), i=1, \ldots, p$. If $F_{i}$ is $p$ times differentiable then the joint density is given by:

$$
c\left(u_{1}, \ldots, u_{p}\right)=\frac{f\left(F_{1}^{-1}\left(u_{1}\right), \ldots, F_{p}^{-1}\left(u_{p}\right)\right)}{\prod_{i=1}^{p} f_{i}\left(F_{i}^{-1}\left(u_{i}\right)\right)}
$$

The copula-GARCH class of models assumes certain parameters are time varying in an autoregressive manner and their distributions are conditional on past information. In the context of this analysis we are interested in modeling the cross sectional dependence between time series data and therefore we employ an adapted version of Sklar's theorem introduced by Patton (2006). The distribution of $Y_{t}$ conditional on $\mathcal{F}_{t-1}=\left\{Y_{1}: 1 \leq t-1\right\}$ is decomposed into its conditional margins and the corresponding conditional copula in the following way:

with

$$
F\left(y \mid \mathcal{F}_{t-1}\right)=C\left\{F_{1}\left(y_{1} \mid \mathcal{F}_{t-1}\right), \ldots, F_{p}\left(y_{p} \mid \mathcal{F}_{t-1}\right) \mid \mathcal{F}_{t-1}\right\}
$$

$$
Y_{i t} \mid \mathcal{F}_{t-1} \sim F_{i}\left(\cdot \mid \mathcal{F}_{t-1}\right), i=1, \ldots, p
$$

Fitting a copula on the unconditional probability integral transform will result in an unconditional copula model for the dependence. In a time series context however, it is necessary to condition on the available past information which first requires the specification of the margins and then the copula that joins the series cross-sectionally. If we define the probability integral transform $U_{i t}=F_{i}\left(Y_{i t} \mid \mathcal{F}_{t-1}\right)$ then the conditional copula of $Y_{t} \mid \mathcal{F}_{t-1}$ is given by $U_{t} \mid \mathcal{F}_{t-1} \sim C\left(\cdot \mid \mathcal{F}_{t-1}\right)$. It is important to note that both the margins and the copula have to be conditional on the same dataset. We use the standard ARMA-GARCH approach to model the univariate distributions by specifying the following general model for each series:

$$
Y_{i t}=\mu_{i}\left(Y_{t-1}\right)+\sigma_{i}\left(Y_{t-1}\right) \epsilon_{i t}
$$

where $\epsilon_{i t} \sim F_{i}(0,1), \forall t$. The conditional copula is fitted on the conditional distribution of the probability integral transform of the standardized residuals constructed as:

$$
\hat{\epsilon}_{i t}=\frac{Y_{i t}-\mu_{i}\left(Y_{t-1}\right)}{\sigma_{i}\left(Y_{t-1}\right)}, i=1,2 \ldots, p
$$

The parametric form of $F_{i}$ has to be able to accommodate thicker than normal tails and possibly an asymmetric shape. For this analysis, we tested different forms of skewed distributions and choose the flexible and thoroughly studied Skew- $t$ form of Lambert and Laurent (2001), for its suitability to our data set.

\section{Dependence Measures}

The literature on multivariate analysis provides a broad array of dependence measures (see Nelsen, 2006, Chapter 5) for an in-depth description. In this study we will focus on rank correlation and tail dependence for 
their specific property of being scale invariant. Linear correlation plays an essential role in finance theory, however it is important to mention that this concept only applies in the context of multivariate elliptical distributions. A detailed discussion of the shortcomings of relying on linear correlation is given by Embrechts et al. (2002). One important property of copulas, namely that the dependence structure is invariant under increasing and continuous transformations of the margins, allows for the specification of the Spearman's rank correlation directly as:

$$
\begin{gathered}
\rho_{S}=\rho\left(F_{1}\left(Y_{1 t}\right), F_{2}\left(Y_{2 t}\right)\right) \\
\hat{\rho}_{S}=\frac{12}{T} \sum_{t=1}^{T} U_{1 t} U_{2 t}-3
\end{gathered}
$$

which is simply the linear correlation of the probability integral transforms. Hence for continuous random variables $\rho_{S}$ is the linear correlation of their unique copula. $\rho_{S}$ is constrained in $[0,1]$ with the boundaries being reached only if the variables are comonotonic or countermonotonic. Despite its appealing characteristics the rank correlation is only a scalar measure that gives an overall indication about the strength and sign of the dependence.

The concept of tail dependence relates to the amount of dependence in the upper or lower quadrant tail of the distribution. The motivation for looking at these coefficients is that they provide measures of extremal dependence and we describe them in terms of limiting conditional probabilities as:

$$
\lambda^{L}=\lim _{q \rightarrow 0} \frac{C(q, q)}{q} \text { and } \lambda^{U}=\lim _{q \rightarrow 1} \frac{1-2 q-C(q, q)}{1-q}
$$

where $C$ is said to have upper tail dependence if $\lambda^{U} \in[0,1]$ and lower tail dependence if $\lambda^{L} \in[0,1]$. If $\lambda^{L}=\lambda^{U}$ then the copula is radially symmetric and if one coefficient is 0 , then the copula is asymptotically independent in the respective tail.

In order to determine whether a time varying conditional copula model is appropriate it is necessary to test for the presence of time-varying dependence. We focus on tests that look for changes in rank correlation. The simplest way to implement this tests is to break the data series at a specific point $t$ and test for $\mathrm{H}_{0}: \rho_{1}=\rho_{2}$ where $\rho_{1}$ and $\rho_{2}$ are the rank coefficients computed for values below and above the break point $t$. The shortcoming of this test is that the break point has to be specified in advance. In order to overcome this difficulty a more intricate test would be to search for the break point by constructing the following statistic:

$$
Q=\max _{t \in\left[t^{L}, t^{U}\right]}\left|\rho_{1, t}-\rho_{2, t}\right|
$$

where:

$$
\rho_{1, t}=\frac{12}{\mathrm{t}} \sum_{i=1}^{t} U_{1 i} U_{2 i}-3 \text { and } \rho_{2, t}=\frac{12}{T-t} \sum_{i=t+1}^{T} U_{1 i} U_{2 i}-3
$$

The significance of the test can be assessed by comparing $Q$ against a critical value derived from a simple iid bootstrap. The interval $\left[t^{L}, t^{U}\right]$ has to be constructed in a way that ensures enough observations are left to estimate pre and post break point rank correlations. Instead of searching for discrete breaks in rank correlation the following test looks for autocorrelation in dependence:

$$
U_{1 t} U_{2 t}=\alpha_{0}+\sum_{i=1}^{p} \alpha_{i} U_{1, t-i} U_{2, t-i}+\varepsilon_{t}
$$

Under the null of a constant conditional copula $\alpha_{i}=0, \forall i \geq 1$. This hypothesis can be tested by using the Ljung-Box test statistic. 


\section{Estimation and Inference}

Estimation for copula models is usually performed via Maximum Likelihood (ML). One may attempt to estimate margins and copula in one single optimization, however splitting the modeling into two steps can yield more insight and allow for more detailed analysis of the different model components.

\section{Constant Copula}

Using parametric models for the conditional distributions and the copula completely specifies the log-likelihood function and the parameters can be estimated as:

$$
\hat{\theta}_{\mathrm{IFM}}=\arg \max _{\theta} \log \mathcal{L}(\theta)
$$

The canonical representation for the multivariate density function in equation (3) permits us to say that a statistical modeling problem for copulas could be decomposed into two steps: identification of the marginal distributions and specification of the appropriate copula function. This approach has been termed inference-functions for margins (IFM) and the parameters are estimated as in:

$$
\begin{gathered}
\hat{\theta}_{1}=\arg \max _{\theta_{1}} \sum_{t=1}^{T} \sum_{i=1}^{p} \log f_{i}\left(Y_{i t} ; \theta_{1}\right) \\
\hat{\theta}_{2}=\arg \max _{\theta_{2}} \sum_{t=1}^{T} \log c\left(F_{1}\left(Y_{1 t} ; \theta_{1}\right), \ldots, F_{p}\left(Y_{2 t} ; \theta_{1}\right) ; \theta_{2}\right)
\end{gathered}
$$

and $\hat{\theta}_{\mathrm{IFM}}=\left[\hat{\theta}_{1}, \hat{\theta}_{2}\right]$ is the parameter vector estimated in stages. In the following we will assume that the regularity conditions of Joe (1997) for parameter asymptotic distribution hold for the multivariate model as well as for all margins. Under these regularity conditions the ML estimator exists and it has the property of being asymptotically normal:

$$
\sqrt{T}\left(\hat{\theta}_{\mathrm{IFM}}-\theta\right) \rightarrow N\left(0, V_{\mathrm{IFM}}\right) \text { for } T \rightarrow \infty
$$

A critical point here is that asymptotic normality does not hold under model misspecification. This implies that the copula likelihood being maximized is not the true likelihood because it is influenced by parameter estimation error in the margins. The naive approach would just use the inverse Hessian of the copula likelihood as an estimate of the covariance matrix for the copula parameters. In order to incorporate the estimation error that propagates from the margins to the copula the following estimator should be used for the covariance matrix:

$$
\begin{gathered}
\hat{V}_{\mathrm{IFM}}=\hat{A}^{-1} \hat{B}\left(\hat{A}^{-1}\right)^{\prime} \\
\hat{A}=\frac{1}{T} \sum_{t=1}^{T} \widehat{H}_{t} \text { and } \hat{B}=\frac{1}{T} \sum_{t=1}^{T} \hat{s}_{t} \hat{s}_{t}^{\prime} \\
\hat{s}_{t}=\left[\hat{s}_{1 t}, \ldots, \hat{s}_{p t}, \hat{s}_{c t}\right] \\
\hat{s}_{i t}=\frac{\partial}{\partial \theta_{1, i}} \log f_{i}\left(Y_{i t} ; \hat{\theta}_{1, i}\right), i=1,2, \ldots, p \\
\hat{s}_{c t}=\frac{\partial}{\partial \theta_{2}} \log c\left(F_{1 t}\left(Y_{i t} ; \hat{\theta}_{1,1}\right), \ldots, F_{p t}\left(Y_{p t} ; \hat{\theta}_{1, p}\right) ; \hat{\theta}_{2}\right)
\end{gathered}
$$

Hessian $\widehat{H}_{t}$ has to be adjusted with the partial derivatives of $\mathcal{L}$ with respect to margins and copula parameters to account for marginal parameter estimation error. 


\section{Time Varying Copula}

We considered the Generalized Autoregressive Score (GAS) model of Creal, Koopman, and Lucas (2013) to model the time-varying parameters for the copula of the standardized residuals. This framework describes the dynamic behavior of dependence by expressing the copula parameters as a function of lagged values and the standardized score of the copula log-likelihood. In order to ensure that the copula parameter remains within the limits of its support this approach requires the modeling of a transformed variable $f_{t}=h(\theta)$ as follows:

$$
f_{t+1}=\omega+\alpha I_{t}^{-1 / 2} s_{t}+\beta f_{t}
$$

where:

$$
\begin{gathered}
s_{t}=\frac{\partial}{\partial \theta} \log c\left(U_{1 t}, U_{2 t} ; \theta_{t}\right) \\
I_{t}=E_{t-1}\left(s_{t} s_{t}^{\prime}\right)
\end{gathered}
$$

The future value of the transformed copula parameter is a function of a constant, its current value and the standardized score of the copula log-likelihood. The use of the score for updating $f_{t+1}$ is intuitive because it defines the steepest direction for improving the model local fit in terms of the likelihood at time $t$. This approach is based on the complete density of observations and provides the natural direction for updating the parameter. A convenient property of the GAS model is that it can be estimated via maximum likelihood.

\section{Goodness-of-Fit Tests}

Goodness-of-Fit (GoF) tests have become standard tools for summarizing model performance. We focus on in-sample Kolmogorov-Smirnov (KS) and Cramér-von Mises (CvM) tests to assess the copula performance. These are two of the most powerful tests available. In the multivariate case these tests rely on the empirical copula as the closest estimate of the true copula. Genest, Rémillard, and Beaudoin (2009) used a simulation study to compare a range of copula GoF tests and conclude that the most powerful is the CvM test applied to the Rosenblatt transforms of the original data. The Rosenblatt transform represents the multivariate version of the probability integral transformation, mapping the original data into a hypercube of mutually independent variables. In the bivariate case, if $Z_{1 t}=U_{1 t}, \forall t$ and $Z_{2 t}=C_{2 \mid 1, t}\left(U_{2 t} \mid U_{1 t} ; \theta\right)$ where $C_{2 \mid 1, t}$ is the distribution of $U_{2 t}$ conditional on $U_{1 t}$, then $Z_{1 t}$ and $Z_{2 t}$ are iid and $U n i f(0,1)$ distributed. The advantage of this transformation is that if the copula is correctly specified then $Z_{t} \sim C_{\text {indep }}$ which allows us to construct the tests as:

$$
\mathrm{KS}=\max _{t}\left|C_{R}\left(Z_{t} ; \hat{\theta}\right)-\hat{C}_{R}\left(Z_{t}\right)\right| \text { and } \mathrm{CvM}=\sum_{t=1}^{T}\left\{C_{R}\left(Z_{t} ; \hat{\theta}\right)-\hat{C}_{R}\left(Z_{t}\right)\right\}^{2}
$$

where:

$$
C_{R}\left(Z_{t} ; \hat{\theta}\right)=\prod_{i=1}^{P} Z_{i t} \text { and } \hat{C}_{R}(z)=\frac{1}{T} \sum_{t=1}^{T} \prod_{i=1}^{p} 1\left\{Z_{i t} \leq z_{i}\right\}
$$

This set-up allows us to test whether the empirical copula, $\hat{C}_{R}(z)$, is statistically different from the independence copula $C_{R}$. Critical values for these tests can be obtained via simulation.

\section{Implementation and Results}

The purpose of this study is to describe the time-varying dependence between the iTraxx Europe Senior Financial and Crossover CDS indices as a measure of contagion between the European corporate and financial sectors. In the first phase, we analyze the dependence pattern between the two series and test for evidence of 
time-varying dependence. In a second phase, we fit both constant and time-varying copula-GARCH model and test for the statistical significance of the estimated parameters. One advantage of the copula-GARCH approach is the possibility to specify the model in stages. The marginal distributions are specified by an ARMA-GARCH model for each univariate time series and then a copula is estimated on the probability integral transforms of the standardized residuals.

The Markiti-Traxx Europe Senior Financial index comprises 25 equally weighted CDSs on investment grade European financial entities whereas the MarkitiTraxx Europe Crossover index comprises 50 equally weighted CDSs on the most liquid sub-investment grade European corporate entities. Given the liquidity and actualized composition of the two indices we believe they are representative indicators of credit risk for their respective sectors. We proceed in analyzing the log-returns of the end-of-day quotations for the two indices with five years maturity. In order to construct a consistent time series we stitched together the log-returns of series 5 to series 19 creating a total of 1,880 observations spanning the time period from March 21, 2006 to June 17, 2013. The return series time span is long enough to include both pre and post crisis market conditions and therefore it is representative of the long run connection between the credit risk of financial and corporate sectors. All data were retrieved from the Bloomberg Database.

Figure 1 depicts the levels of the two indices during the analyzed period. There is clearly more variability in the crossover index explained by its composition of sub-investment grade CDSs that react more vigorously to changing economic conditions. Although not as volatile, the financial index shows a steady upward trend indicating increasing risk in the financial sector. The support provided by the European authorities alleviated the risks for financial institutions and kept the index relatively stable at around 200bp for the past two years.

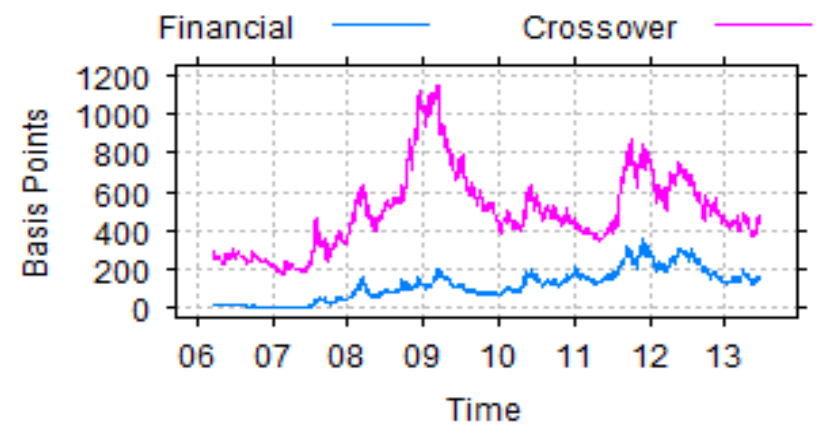

Figure 1. iTraxx financial and crossover index levels (left panel).

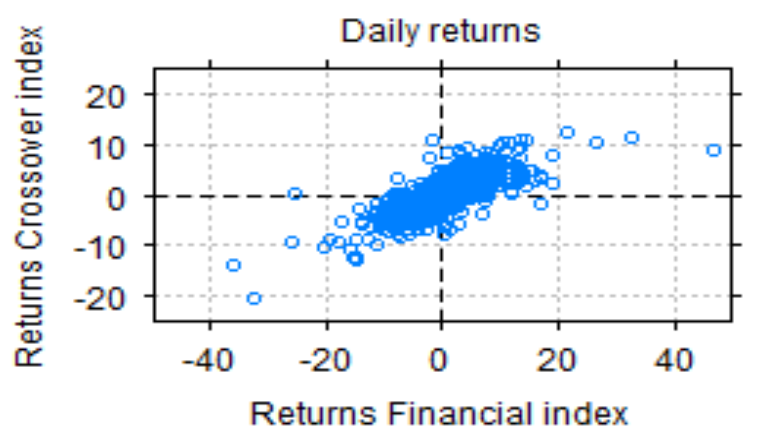

Figure 2. Daily returns of the two indices (right panel). 
The summary statistics presented in Table 1 indicate that the returns of the two indices have fat tails and possibly skewed distributions. Both linear and rank correlation coefficients indicate that risks in the two sectors are dependent while the scatter plot in Figure 2 provides a visual interpretation of the direction and strength of dependence. This pattern is well-suited for elliptical as well as Archimedean copula classes. Starting from 2010, the correlation between the two indices has increased, providing an indication of possible credit risk contagion effects between the two sectors.

Table 1

Summary Statistics for the Returns of the iTraxx Financial and Crossover Indices

\begin{tabular}{lcc}
\hline Indicator & Financial & Crossover \\
\hline Mean & 0.1083 & -0.0348 \\
Std. dev. & 4.7961 & 2.8720 \\
Skewness & 0.3146 & 0.0488 \\
Kurtosis & 10.7897 & 3.1590 \\
Corr. Pearson/Spearman & $0.7804 / 0.7907$ & \\
\hline
\end{tabular}

Applying an ARMA-GARCH process to the return series of the two indices removes the temporal correlation but preserves the cross-sectional dependence. The order for the mean equation was determined by comparing the BIC for ARMA models of orders up to five. An AR(1) process was optimal for both series and this decision has also been confirmed by the partial autocorrelation function of the squared log-returns. Then, we used the squared residuals from the mean equation to test for conditional heteroscedasticity. The Ljung-Box test applied on the first 12 squared log-returns revealed $p$-values very close to zero which motivates the introduction of a conditional variance equation. Empirical studies have shown that asset returns may have fat tails and skewed distributions. To handle these characteristics, we used the skewed version of the Student- $t$ distribution proposed by Lambert and Laurent (2001), for its suitability to ARCH processes. Therefore, in the framework described by equation (4), we filtered the data by fitting an AR(1)-GARCH(1,1)-Skew- $t$ model on each series in order to remove the autocorrelation and construct the standardized residuals as in equation (6). In addition to autoregressive models we tested for cross-correlation by regressing one series on up to five lags of the other series but the coefficients came out not significant, at 5\% level. This finding together with the shape of the scatter plot in Figure 2 is a strong indication that the two series are not linearly correlated but dependent.

The copula chosen to describe the tail dependence must provide non-zero tail dependence in the specific tail on which is to be used. According to Capéràa, Fougéres, and Genest (2000), the Gumbel copula is also an extreme value copula and Archimedean copulas that belong to a domain of attraction are necessarily in the domain of attraction of the Gumbel copula. Given its properties, the Gumbel copula is a natural choice regarding tail dependence estimation. Table 2 presents the tail dependence coefficients determined according to equation (8) by fitting the Gumbel copula to the probability integral transforms of the standardized residuals. Confidence intervals (CI) are determined using an iid bootstrap simulation. Even though the upper and lower tail dependence coefficients are different, with CI overlapping, the $p$-value for $\mathrm{H}_{0}: \lambda^{L}=\lambda^{U}$ is 0.4416 . The first conclusion stemming from the results presented in Table 2 is that we fail to reject the null of symmetric dependence. In addition, none of the confidence interval include zero which leads us to the second conclusion that both tail dependence coefficients are statistically significant. The economic interpretation of these two conclusions is that extreme credit events in one sector cannot occur without affecting the other. That is, the 
credit risks of the two sectors are linked regardless of the direction, providing statistical evidence that supports the existence of contagion effects.

Table 2

Estimates of Tail Dependence Using Gumbel Copula

\begin{tabular}{lll}
\hline Estimate/Confidence interval & Lower tail dependence & Upper tail dependence \\
\hline$\lambda$ & 0.4346 & 0.3325 \\
$90 \% \mathrm{CI}$ & {$[0.3368,0.5514]$} & {$[0.2151,0.4298]$} \\
\hline
\end{tabular}

Our reason for looking at rank correlation is because it provides information on the sign of the dependence and provides valuable indication on which types of copulas to use. The rank correlation of standardized residuals, calculated according to equation (7), is 0.7779 and using a simple iid bootstrap we computed a $90 \%$ CI of [0.7597, 0.7952]. Unlike elliptical copulas, Archimedean copulas can only capture positive dependence and having a statistically significant positive rank correlation justifies the application of these copulas.

Under the assumption of constant dependence we use four copulas to model the full dependence structure. The Gaussian copula has become the standard tool in dependence modeling and we use it as a benchmark. The Student- $t$ copula is motivated by the shape of the scatter plot of returns and its ability to capture tail dependence in both tails. The Gumbel and Clayton copulas are representative for the Archimedean class and provide asymmetric contour diagrams. Given the larger lower tail dependence parameter the Gumbel copula was used in the rotated form. In the multi-stage copula-GARCH framework copula estimation is straightforward because the optimization is performed over one or two parameters. Given the large sample of our data set we relied on multi-stage IFM estimation. Table 3 presents the log-likelihood, parameter estimates and standard errors for the constant copulas of the standardized residuals. The Student- $t$ copula performed best as indicated by the largest log-likelihood value. This finding has two explanations. First, the characteristics of this copula are closest to the pattern exhibited by our data set and in particular it can capture both lower and upper tail dependency. Second, it has two parameters benefiting from the flexibility of a parameter that controls exactly the thickness in the tails. Even though the Gaussian copula cannot capture tail dependence it provided the second best fit. This is an indication that tail events have a low weight in setting the strength of the dependence. The rotated Gumbel copula performed close to the benchmark set by the Gaussian. This stems from the fact that our data set has both lower and upper tail dependency and this copula can only properly describe one tail at a time. Clayton copula proved inappropriate for this data set. The naive standard errors are significantly lower than the correct multi-stage IFM errors giving a false impression of consistency. This is because the naive method ignores the parameter estimation error in the marginal distributions.

Table 3

Parameter Estimates and Standard Errors for Constant Copula Models

\begin{tabular}{lcccc}
\hline & \multicolumn{2}{c}{ Log $\mathcal{L}$} & & \multicolumn{2}{l}{ Standard errors } \\
\cline { 4 - 5 } & & & Naive & IFM \\
\hline Gaussian & 899.7 & 0.7848 & 0.0071 & 0.0193 \\
Student- $t \theta$ & 948.9 & 0.7951 & 0.0082 & 0.0152 \\
$y^{-1}$ & & 0.1821 & 0.0261 & 0.1221 \\
Rotated Gumbel & 889.1 & 2.2773 & 0.0433 & 0.1044 \\
Clayton & 727.8 & 1.7982 & 0.0604 & 0.1737 \\
\hline
\end{tabular}


Figure 3 presents a time series plot of a 60-day rolling rank correlation for the standardized residuals together with $90 \%$ bootstrap point-wise CI. This graph shows that the rank correlation was significantly lower in the period before the crisis. Since the beginning of 2008 this indicator hovered around 0.8 , confirming the strength of the link between the credit risks of the two sectors. Having such a different pattern of correlation between the pre and post crisis periods gives us a reason to believe that the conditional dependence structure may also vary through time.

In addition to the visual interpretation it is important to test for the presence of time-varying dependence and therefore we applied the two break tests and the ARCH test. The $p$-values for the break tests and for the Q test in equation (9) are presented on the left side of Table 4. Using the information provided by Figure 3 we have chosen three break points at $t / T \in\{0.2,0.5,0.8\}$ which correspond to the dates $\{2007-09-31$, 2009-11-03, 2011-11-29\}. The $p$-values reject the null hypothesis of a constant rank correlation and provide statistical proof of a possible break point at the end of September 2007. On the right side, Table 4 presents the $p$-values of the Ljung-Box statistic calculated for the ARCH test in equation (11). Given the very low $p$-values at any lag we can reject the null of constant conditional copula. These results together with the conclusions drawn from Figure 3 provide the prerequisites for using a time varying copula model.

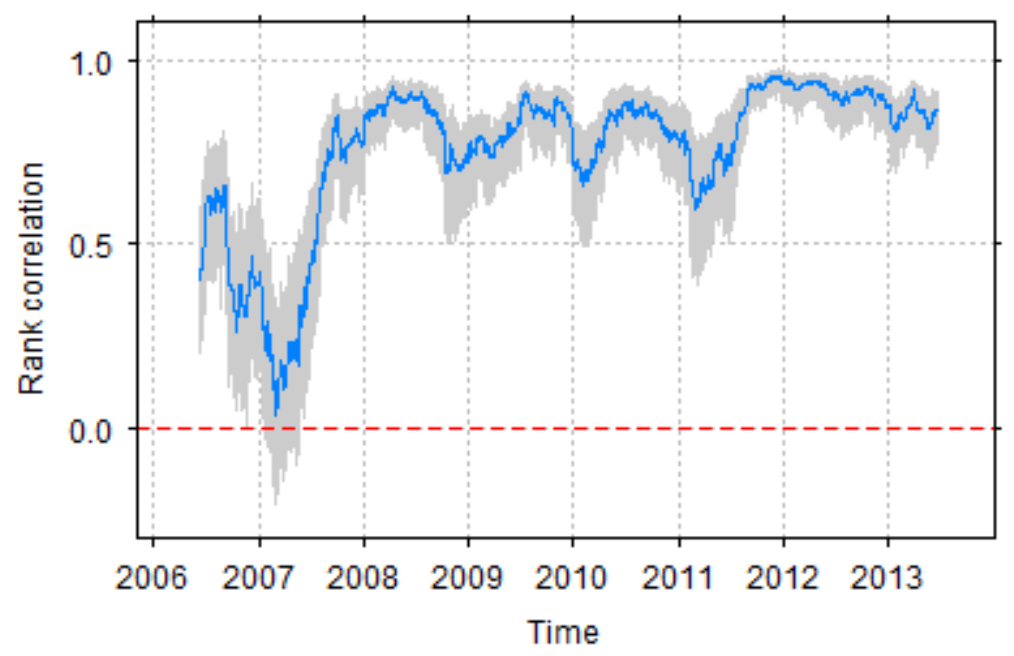

Figure 3. Sixty-day rolling window rank correlation.

Table 4

Results for Time Varying Dependence Tests

\begin{tabular}{lllllllll}
\hline & Break test & \multicolumn{5}{l}{ ARCH test } \\
\cline { 2 - 4 } & 0.2 & 0.5 & 0.8 & Anywhere & & AR(1) & AR(5) & AR(10) \\
\hline$p$-value & 0.0015 & 0.2234 & 0.6177 & 0 & 0 & 0.0031 & 0.0058 \\
\hline
\end{tabular}

We considered the Student $t$ GAS and rotated Gumbel GAS copulas to model the time-varying copula parameters. To ensure the copula parameter remains within the accepted range we adopted the transformations proposed by Patton (2012). For the Gumbel copula the function $\theta_{t}=1+\exp \left(f_{t}\right)$ is used to ensure the parameter remains greater than 1 . In the Student- $t$ copula case, the degree of freedom parameter is kept constant and the correlation parameter is transformed as in $\theta_{t}=\left(1-\exp \left(-f_{t}\right)\right) /\left(1+\exp \left(-f_{t}\right)\right)$ to ensure it lies ininterval $(-1,1)$. The parameter estimates for these two models together with their standard errors are presented in Table 5. 
Table 5

Parameter Estimates and Standard Errors for Time Varying Copula Models

\begin{tabular}{|c|c|c|c|c|c|}
\hline & \multirow{2}{*}{$\log \mathcal{L}$} & \multirow{2}{*}{ Parameters } & & \multicolumn{2}{|c|}{ Standard errors } \\
\hline & & & & Naive & IFM \\
\hline \multirow{3}{*}{$\begin{array}{l}\text { Gumbel } \\
\text { GAS }\end{array}$} & 971.1 & $\omega$ & 0.0017 & 0.0008 & 0.0123 \\
\hline & & $\alpha$ & 0.0582 & 0.0137 & 0.4544 \\
\hline & & $\beta$ & 0.9944 & 0.0019 & 0.1201 \\
\hline \multirow{4}{*}{$\begin{array}{l}\text { Student- } t \\
\text { GAS }\end{array}$} & 1047.6 & $\omega$ & 0.0099 & 0.0014 & 0.2401 \\
\hline & & $\alpha$ & 0.0531 & 0.0094 & 0.5469 \\
\hline & & $\beta$ & 0.9957 & 0.0001 & 0.3346 \\
\hline & & $v^{-1}$ & 0.0866 & 0.0158 & 0.0525 \\
\hline
\end{tabular}

According to our priori-expectations the log-likelihoods for the time-varying copulas are significantly greater than the ones for constant copulas. This indicates that the GAS models are better at describing the dependence than their constant counterparts. The $\beta$ parameter in equation (16) is very close to 1 which indicates a high temporal dependence in copula parameters. Even though the $\alpha$ parameter is low, it is statistically significant, which indicates that the future copula parameters are dependent on past information as described by the score of copula likelihood function. The degrees of freedom parameter of the Student- $t$ copula is greater in the time-varying model than in the constant model. This result is consistent with previous findings in volatility literature. As in the constant case, the naive standard errors are smaller than the IFM errors that correctly include the parameter estimation error in the margins. This finding is also in line with theoretical guidelines.

The results outlined above allow us to conclude that the credit risk dependence is indeed significant and time-varying. We may conclude that the statistical models employed provide sufficient evidence in support of the existence of credit risk contagion between the corporate and financial sectors, at European level.

In the absence of parameter estimation error GoF test statistics follow some known distribution. However, known parametric distributions are not applicable here because KS and CvM depend on $\hat{\theta}$, that is they are affected by estimation error both in marginal distributions and copula. This is the reason why simulation represents the most suitable method for determining $p$-values for KS and CvM tests. The following simulation procedure is straight forward and involves sampling and estimation of both margins and copula: (1) estimate the model on the original data to obtain $\hat{\theta}$ and compute the GoF statistics; (2) simulate a sample of length $T$ from the model using the estimated parameter $\hat{\theta}$; (3) re-estimate the model on the sample data to obtain $\hat{\theta}_{n}$ and compute the GoFn statistics; and (4) repeat steps (2)-(3), $N$ times and compute the $p$-values as: $p_{N}=$ $\sum_{n=1}^{N} 1\left\{\mathrm{GoF}_{n} \geq \mathrm{GoF}\right\} / N$.

Following this simulation procedure we generated the $p$-values for the GoF tests computed on the Rosenblatt transform of the original data. The results are presented in Table 6. Low $p$-values are evidence against the null that the tested copula is the true unknown copula. Student- $t$ and Gumbel copulas pass the test both in the constant and time-varying set-up. This result comes in support of the permanent character of the connection between the credit risk of the two sectors. The validity of both the time-varying and constant copula models indicates that even though the degree of dependence varies in time it has been statistically significant throughout the analyzed period. Therefore, we may conclude that GoF tests provide conclusive evidence supporting credit risk contagion between the two sectors. 
Table 6

p-values for Goodness-of-Fit Tests of Both Constant and Time-Varying Copula Models

\begin{tabular}{|c|c|c|c|c|c|c|}
\hline & \multicolumn{4}{|c|}{ Constant } & \multicolumn{2}{|l|}{ Time varying } \\
\hline & Normal & Student- $t$ & Gumbel & Clayton & Gumbel GAS & Student $-t$ GAS \\
\hline$\overline{\mathrm{KS}}$ & 0.02 & 0.15 & 0.38 & 0.08 & 0.22 & 0.18 \\
\hline CvM & 0.03 & 0.21 & 0.16 & 0.09 & 0.18 & 0.23 \\
\hline
\end{tabular}

\section{Conclusions}

We propose a multi-stage time-varying copula-GARCH model to describe the credit risk dependence between corporate and financial sectors at European level. The analysis indicates a symmetric dependence structure with statistically significant tail dependence parameters. We bring arguments supporting the time-varying dependence and model this pattern with the Student- $t$ and Gumbel GAS models. The statistical significance of our results allows us to conclude that credit risk dependence exists which supports the hypothesis of credit risk contagion. The GoF tests indicate that both time-varying and constant conditional copula models are able to capture the credit risk dependence. The Student- $t$ and Gumbel copula models, validated by the GoF tests, provide conclusive evidence supporting the contagion effects between the corporate and financial sectors.

\section{References}

Capéràa, P., Fougéres, A. L., \& Genest, C. (2000). Bivariate distributions with given extreme value attractor. Journal of Multivariate Analysis, 72(1), 30-49.

Choroś, B., Ibragimov, R., \& Permiakova, E. (2010). Copula estimation. In F. Durante, W. Härdle, \& T. Rychlik (Eds.), Copula theory and its applications (pp. 77-91). New York: Springer.

Creal, D., Koopman, S. J., \& Lucas, A. (2013). Generalized autoregressive score models with applications. Journal of Applied Econometrics, 28(5), 777-795.

Embrechts, P., McNeil, A., \& Straumann, D. (2002). Correlation and dependence in risk management: Properties and pitfalls. In M. A. S. Dempster (Ed.), Risk management: Value at risk and beyond (pp. 176-223). New York: Cambridge University Press.

Genest, C., Rémillard, B., \& Beaudoin, D. (2009). Goodness-of-fit tests for copulas: A review and a power study. Insurance: Mathematics and economics, 44(2), 199-213.

Joe, H. (1997). Multivariate models and dependence concepts: Monographs on statistics and applied probability series. London: Chapman \& Hall.

Lambert, P., \& Laurent, S. (2001). Modelling financial time series using GARCH-type models with a skewed student distribution for the innovations. Discussion paper 0125, Institut de Statistique, Université Catholique de Louvain, Louvain-la-Neuve, Belgium.

McNeil, A. J., Frey, R., \& Embrechts, P. (2005). Quantitative risk management: Concepts, techniques, and tools. New Jersey: Princeton University Press.

Nelsen, R. B. (2006). An introduction to copulas. New York: Springer.

Patton, A. J. (2006). Modelling asymmetric exchange rate dependence. International Economic Review, 47(2), 527-556.

Patton, A. J. (2009). Copula-based models for financial time series. In T. G. Andersen, R. A. Davis, J. P. Krei $\beta$, \& T. Mikosch (Eds.), Handbook of financial time series (pp. 767-785). Berlin: Springer.

Patton, A. J. (2012). Copula methods for forecasting multivariate time series. In G. Elliott, \& A. Timmermann (Eds.), Handbook of economic forecasting (pp. 283-360), North Holland.

Rémillard, B. (2010). Goodness-of-fit tests for copulas of multivariate time series. Retrieved from http://ssrn.com/abstract=1729982 